Check for updates

Cite this: Chem. Sci., 2018, 9, 7405

๑ All publication charges for this article have been paid for by the Royal Society of Chemistry

Received 5th June 2018

Accepted 7th August 2018

DOI: $10.1039 / c 8 s c 02471 e$

rsc.li/chemical-science

\section{High electrical conductivity and high porosity in a Guest@MOF material: evidence of TCNQ ordering within $\mathrm{Cu}_{3} \mathrm{BTC}_{2}$ micropores $\uparrow$}

\author{
Christian Schneider, (iD) a Dardan Ukaj, (DD ${ }^{a}$ Raimund Koerver, ${ }^{b}$ A. Alec Talin, ${ }^{c}$ \\ Gregor Kieslich, (D) a Sidharam P. Pujari, (D) d Han Zuilhof, (D) de Jürgen Janek, (D) b \\ Mark D. Allendorf (D) ${ }^{* c}$ and Roland A. Fischer (D) *a
}

\begin{abstract}
The host-guest system TCNQ@Cu $3 \mathrm{BTC}_{2}(\mathrm{TCNQ}=$ 7,7,8,8-tetracyanoquinodimethane, $\mathrm{BTC}=1,3,5$ benzenetricarboxylate) is a striking example of how semiconductivity can be introduced by guest incorporation in an otherwise insulating parent material. Exhibiting both microporosity and semiconducting behavior such materials offer exciting opportunities as next-generation sensor materials. Here, we apply a solvent-free vapor phase loading under rigorous exclusion of moisture, obtaining a series of the general formula $x \mathrm{TCNQ} \mathrm{CCu}_{3} \mathrm{BTC}_{2}(0 \leq x \leq 1.0)$. By using powder $\mathrm{X}$-ray diffraction, infrared and $X$-ray absorption spectroscopy together with scanning electron microscopy and porosimetry, we provide the first structural evidence for a systematic preferential arrangement of TCNQ along the (111) lattice plane and the bridging coordination motif to two neighbouring $\mathrm{Cu}$-paddlewheels, as was predicted by theory. For 1.0TCNQ $\mathrm{CCu}_{3} \mathrm{BTC}_{2}$ we find a specific electrical conductivity of up to $1.5 \times 10^{-4} \mathrm{~S} \mathrm{~cm}^{-1}$ whilst maintaining a high BET surface area of $573.7 \mathrm{~m}^{2} \mathrm{~g}^{-1}$. These values are unmatched by MOFs with equally high electrical conductivity, making the material attractive for applications such as super capacitors and chemiresistors. Our results represent the crucial missing link needed to firmly establish the structure-property relationship revealed in $\mathrm{TCNQ} \mathrm{CCu}_{3} \mathrm{BTC} \mathrm{C}_{2}$, thereby creating a sound basis for using this as a design principle for electrically conducting MOFs.
\end{abstract}

\section{Introduction}

The development of electrically (semi-)conductive metalorganic frameworks (MOFs) is of great scientific and technological interest, offering the opportunity of making electronic devices with permanent microporosity., ${ }^{1,2}$ MOFs are supramolecular coordination complexes composed of metal ions or clusters that are linked by polydentate organic ligands to form 2D or 3D frameworks with accessible porosity. ${ }^{3}$ By combining both long-range order found in inorganic semiconductors and high chemical tunability found in organics, semiconducting

\footnotetext{
${ }^{a}$ Department of Chemistry, Technical University Munich, Lichtenbergstrasse 4, 85748 Garching, Germany. E-mail: roland.fischer@tum.de

${ }^{b}$ Institute of Physical Chemistry, Center for Materials Research (ZFM), Justus-LiebigUniversity Giessen, Heinrich-Buff-Ring 17, 35392 Giessen, Germany

'Sandia National Laboratories, Livermore, CA 94551, USA. E-mail: mdallen@sandia.gov ${ }^{d}$ Laboratory of Organic Chemistry, Wageningen University \& Research, Stippeneng 4, 6708 WE Wageningen, The Netherlands

${ }^{e}$ School of Pharmaceutical Sciences and Technology, Tianjin University, 92 Weijin Road, Tianjin, P. R. China

$\dagger$ Electronic supplementary information (ESI) available: Experimental details, PXRD patterns, BET surface area analysis, IR spectra, thermogravimetric analysis, current-voltage curves, Auger electron spectroscopy, SEM images, XPS spectra, elemental analysis. See DOI: 10.1039/c8sc02471e
}

MOFs are unique among the different classes of conducting materials. In principle, the deep understanding of coordination chemistry and crystal engineering enables rational design of MOF systems at a level that is difficult to achieve in other (particularly non-crystalline) materials. ${ }^{4,5}$ However, most MOFs are insulators, originating from the rather ionic coordination bonds of metal carboxylates that tend to suppresses charge transfer between the metal node and linker. ${ }^{6}$ Typically, design strategies for electrically conductive MOFs focus on the use of organic linkers that form coordination bonds with improved orbital delocalization between metal and ligand to facilitate charge transfer, and on the use of metal ions with high-energy valance electrons such as $\mathrm{Cu}^{2+}$ and $\mathrm{Fe}^{2+} .6,7$

In 2014, some of us applied a radically new approach to impart electrical conductivity to MOFs by incorporating the redox-active molecule TCNQ (7,7,8,8-tetracyanoquinodimethane) in the pores of $\mathrm{Cu}_{3} \mathrm{BTC}_{2}$ (also known as HKUST-1; BTC $=1,3,5$-benzenetricarboxylate). ${ }^{8}$ Since then, this striking observation has been confirmed by other groups; ${ }^{9,10}$ however, the detailed nature of the host-guest complex and the conductivity mechanism are mainly unsolved. It is proposed that TCNQ bridges two $\mathrm{Cu}$ dimer units via two geminal nitrile groups, which is strongly supported by both theory and spectroscopic data. ${ }^{\mathbf{8}, 11}$ Initial measurements of the Seebeck 
coefficient revealed that holes are the majority charge carriers, ${ }^{12}$ and an underlying super-exchange mechanism was proposed. ${ }^{13}$ Neumann et al. reported calculations supporting this hypothesis, but required the majority carrier to be electrons, which is in apparent conflict with the abovementioned Seebeck measurements. ${ }^{10}$ Furthermore, detailed and clear structural evidence for the bridging binding motif has not been obtained, despite intense research efforts. A factor confounding interpretation of prior results is the use of solution-phase techniques for the infiltration of TCNQ into the host framework, during which solvent molecules and TCNQ compete for free $\mathrm{Cu}$ (II) coordination sites. This likely impedes long-range ordering of TCNQ molecules in the framework and disrupts diffusion pathways for the guest molecule. Moreover, TCNQ is easily reduced (redox potential of $+0.2 \mathrm{~V} v s$. $\mathrm{Ag} / \mathrm{AgCl}$ ), ${ }^{\mathbf{1 4 , 1 5}}$ and in turn can react with the organic solvent and/or water molecules, preventing full control of the oxidation state of TCNQ molecules adsorbed in the $\mathrm{Cu}_{3} \mathrm{BTC}_{2}$ framework. In other words, the material obtained via liquid phase infiltration lacks rigorous experimental evidence possibly needed for a profound understanding of the conductivity mechanism due to a wider parameter space of compositional and structural characteristics. Is there a single type of TCNQ guest or is there a range of guest species including neutral and charged and how relevant is a homogenous long range ordering vs. short range (nanodomains) or even random and disordered distribution of TCNQ? Is the material phase pure or are their impurities to be considered? What is the role of water and solvent coordinated to $\mathrm{Cu}$ centers that are not occupied by TCNQ and do traces of physisorbed water or other protic impurities (e.g. methanol, ethanol) influence the conductivity of the material? Pristine $\mathrm{Cu}_{3} \mathrm{BTC}_{2}$ samples (bulk as well as thin film) are known to exhibit significant amounts of $\mathrm{Cu}(\mathrm{I})$ defect sites. Do these sites play a role when the material is loaded with redox non-innocent guests such as TCNQ? This more complex scenario of questions prompted us to investigate preparative concepts for loading $\mathrm{Cu}_{3} \mathrm{BTC}_{2}$ with TCNQ.

Recently, D'Alessandro and co-workers applied a vacuum vapor-phase infiltration (VPI) for the incorporation of TCNQ into $\mathrm{Cu}_{3} \mathrm{BTC}_{2} \cdot{ }^{16}$ In their approach, however, infiltrated samples were subsequently washed with solvent to remove excess, uninfiltrated TCNQ. Furthermore, samples were treated under ambient conditions, compromising efforts to keep the hostguest system free from solvent and moisture. Our study builds on their important work, applying an optimized VPI method with rigorous exclusion of moisture and solvent contaminations. Following this new protocol, we obtain samples with precise stoichiometry, having the general formula $x$ TCNQ@ $\mathrm{Cu}_{3} \mathrm{BTC}_{2}(0 \leq x \leq 1)$, that are devoid of complicating factors associated with liquid-phase infiltration. The materials were characterized by powder X-ray diffraction (PXRD), infrared (IR) and X-ray photoelectron spectroscopy (XPS) and electrical conductivity measurements, with all measurements strictly conducted under inert conditions. Strikingly, we find evidence of long-range order for TCNQ@ $\mathrm{Cu}_{3} \mathrm{BTC}_{2}$ samples with highloadings. Despite the observation that minor amounts of $\mathrm{Cu}$ (TCNQ) form as byproduct on the surface of $\mathrm{Cu}_{3} \mathrm{BTC}_{2}$ crystals, we find a relatively high electrical conductivity of these samples. Moreover, by avoiding the use of solvent and careful sample handling, our VPI method yields high permanent porosity as well - more than five times higher than achieved previously using liquid-phase infiltration ${ }^{8}$ - demonstrating that high electrical conductivity and large pore volume in MOFs can be compatible.

\section{Results and discussion}

The physical mixture of activated, desolvated $\mathrm{Cu}_{3} \mathrm{BTC}_{2}$ and TCNQ (ground together in a mortar) was annealed under vacuum in a glass ampule and heated for 3 days at $180{ }^{\circ} \mathrm{C}$ to yield the host-guest complex. Using the VPI approach, we produced a series of samples of the general formula $x$ TCNQ@ $\mathrm{Cu}_{3} \mathrm{BTC}_{2}\left(x=n(\mathrm{TCNQ}) / n\left(\mathrm{Cu}_{3} \mathrm{BTC}_{2}\right)\right.$ with $x=0.1$, $0.2, \ldots, 1.0)$ with precisely defined guest loadings by varying the amount of TCNQ with respect to a fixed amount of $\mathrm{Cu}_{3} \mathrm{BTC}_{2}$. Even though a loading of $x=1.5$ would be necessary to saturate all open $\mathrm{Cu}$ sites, unreacted, crystalline TCNQ was observed by powder X-ray diffraction (PXRD) and scanning electron microscopy (SEM) for samples with $x>1.0$. This is in accordance with the maximum loading capacity of two TCNQ per large pore (one TCNQ per formula unit $\mathrm{Cu}_{3} \mathrm{BTC}_{2}, x=1$ ) predicted by theory. ${ }^{8}$ The PXRD data of the loaded series of $x$ TCNQ@ $\mathrm{Cu}_{3}$ $\mathrm{BTC}_{2}$ (Fig. 1) show that the overall crystal structure of $\mathrm{Cu}_{3} \mathrm{BTC}_{2}$ is retained upon TCNQ infiltration. The largest change observed is in the intensity of the (111) reflection at $2 \theta=5.82^{\circ}$, which increases monotonically with the loading amount of TCNQ (highlighted in Fig. 1). Additionally, new reflections appear at higher angles, of which only the weak reflection at $15.77^{\circ}$ matches with the principal reflection of a potential byproduct,

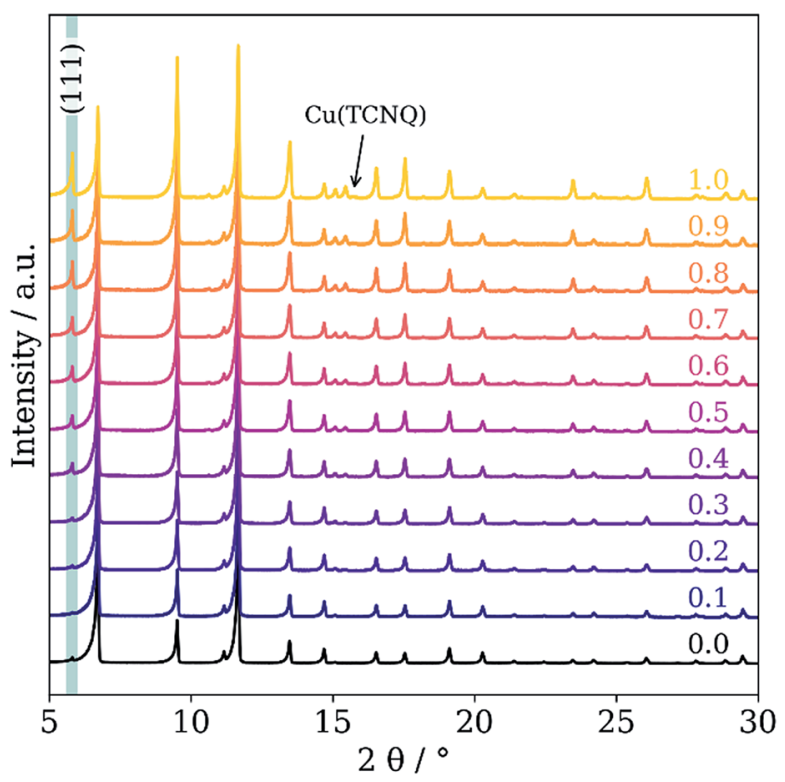

Fig. 1 PXRD patterns of pristine $\mathrm{Cu}_{3} \mathrm{BTC}_{2}$ (black) and the concentration series of $T C N Q$-loaded $\mathrm{Cu}_{3} B T C_{2}$. TCNQ loadings are given above each pattern as $x$ in $x T C N Q Q_{C C_{3} B T C}$. Traces of $\mathrm{Cu}(T C N Q)$ phase I can be found at high TCNQ loadings. 
i.e. $\mathrm{Cu}(\mathrm{TCNQ})$ phase I ( $c f$. Fig. $\mathrm{S} 1 \dagger)$. The increase of the (111) reflection has previously been interpreted as an indicator for chemisorption of guests at the open $\mathrm{Cu}$ sites, ${ }^{17}$ and is here indicative of the incorporation of TCNQ into $\mathrm{Cu}_{3} \mathrm{BTC}_{2}$. Since the modified VPI method excludes any intensity changes due to solvent incorporation, this finding is consistent with the suggested bridging binding mode of TCNQ, as the $\mathrm{Cu}$ atoms of the paddlewheel nodes direct the planar TCNQ molecule into the (111) lattice plane (Fig. 2). Nevertheless, there will be disordered TCNQ inside of the pores considering that there are two types of large pores in $\mathrm{Cu}_{3} \mathrm{BTC}_{2}$ and only one features open $\mathrm{Cu}$ sites pointing to the center of the pore, i.e. are available for coordination of TCNQ. Furthermore, we observed weak reflections with increasing $x$ in $x \mathrm{TCNQ} @ \mathrm{Cu}_{3} \mathrm{BTC}_{2}$ that are not in agreement with the parent face-centered cubic cell of $\mathrm{Cu}_{3} \mathrm{BTC}_{2}$, e.g. at $10.65^{\circ}, 15.44^{\circ}$ and $18.20^{\circ}$. However, they are consistent with a primitive unit cell of parent $\mathrm{Cu}_{3} \mathrm{BTC}_{2}$ having similar lattice parameters, therefore pointing to decreased lattice symmetry for the infiltrated MOF. For example, a Pawley profile fit with reduced symmetry $(P n \overline{3} m)$ and maintaining the same lattice parameters as the parent $\mathrm{Cu}_{3} \mathrm{BTC}_{2}$ can account for all additional reflections (Fig. S4†). A doubling of the unit cell parameters while keeping the face-centered symmetry could similarly lead to a good profile fit, which might be in better chemical agreement, reflecting the different pores with and without available $\mathrm{Cu}$ sites. Determining the origin of these reflections requires additional structural analysis that is complex and extends significantly beyond the scope of the present report. However, this finding is intriguing, pointing to an ordering phenomenon related to TCNQ molecules and a subsequent symmetry reduction of the host-guest system that has not been previously observed. Based on the PXRD measurement, we therefore conclude that our modified VPI method leads to incorporation of TCNQ into $\mathrm{Cu}_{3} \mathrm{BTC}_{2}$ with the potential formation of a supercell.

In order to probe the porosity as function of $x$ in $x$ TCNQ@ $\mathrm{Cu}_{3} \mathrm{BTC}_{2}$, we performed nitrogen adsorption experiments. As expected, a decrease of the Brunauer-Emmett-Teller (BET) surface area is observed, from $1833.0 \mathrm{~m}^{2} \mathrm{~g}^{-1}$ for pristine $\mathrm{Cu}_{3} \mathrm{BTC}_{2}$ to $573.7 \mathrm{~m}^{2} \mathrm{~g}^{-1}$ for 1.0TCNQ@ $\mathrm{Cu}_{3} \mathrm{BTC}_{2}$ (Fig. 3), about

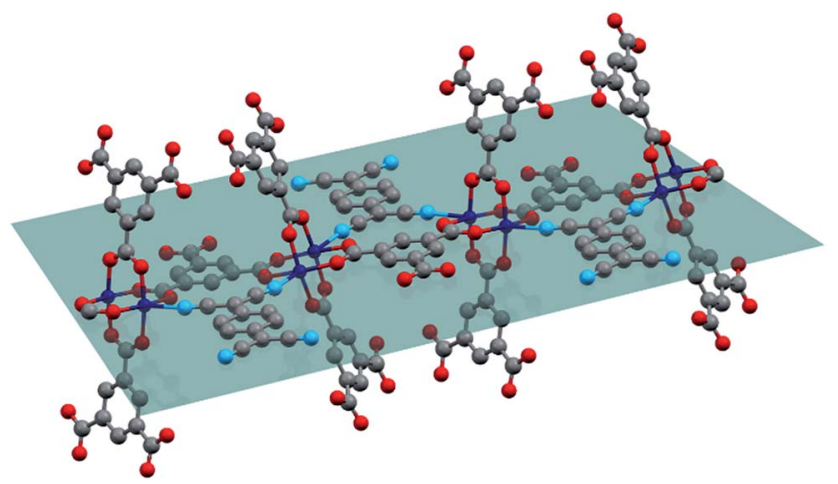

Fig. 2 Schematic representation of TCNQ binding to the apical position of two neighbouring $\mathrm{Cu}$ paddlewheels directing the planar molecule into the (111) lattice plane (tile). For better visualization, the cubic symmetry is not applied. $\mathrm{Cu}, \mathrm{C}, \mathrm{O}$, and $\mathrm{N}$ atoms are depicted in blue, grey, red, and light blue, respectively. two thirds of the initial BET surface area. In addition, the decrease is linear with TCNQ loading, providing additional evidence for the incorporation of the guest molecule into the framework. Importantly, the decrease cannot be explained by pore blocking or a simple adsorption of TCNQ at the crystal surface. For instance, the BET surface area of a physical mixture of both components for 1.0TCNQ@ $\mathrm{Cu}_{3} \mathrm{BTC}_{2}$ would still amount to $1370.4 \mathrm{~m}^{2} \mathrm{~g}^{-1}$ (see detailed discussion in ESI $\dagger$ ). It is further important to note that our measured surface areas considerably exceed those for TCNQ-loaded $\mathrm{Cu}_{3} \mathrm{BTC}_{2}$ samples reported in the literature. For example, the VPI 0.5TCNQ@ $\mathrm{Cu}_{3} \mathrm{BTC}_{2}$ sample exhibits a surface area of $1145 \mathrm{~m}^{2} \mathrm{~g}^{-1}$, whereas the surface area of the material with the same loading synthesized via liquid phase infiltration is reported as $214 \mathrm{~m}^{2} \mathrm{~g}^{-1}$, which is likely due to residual adsorbed water or solvent. ${ }^{8}$

The microstructure and phase homogeneity of the TCNQloaded samples was further investigated using SEM (Fig. 4). Nanowire-like structures were discovered on the external surface of the TCNQ-loaded MOF crystallites; the amount and dimensions of these increase with the amount of TCNQ employed during the VPI. To determine the chemical nature of the nanowires, scanning Auger electron microscopy was performed. These data show a homogenous distribution of $\mathrm{Cu}, \mathrm{C}$, and $\mathrm{N}$ (Fig. S13 $\dagger$ ). In combination with the characteristic morphology ${ }^{18}$ and the reflection at $15.77^{\circ}$ in the diffraction pattern, the nanowires were identified as phase I of $\mathrm{Cu}$ (TCNQ). For the formation of $\mathrm{Cu}$ (TCNQ) to occur, both TCNQ and $\mathrm{Cu}$ (II) must be reduced to $\mathrm{TCNQ}^{-}$and $\mathrm{Cu}(\mathrm{I})$, presumably involving an oxidation (decarboxylation) of the BTC linker under the conditions of the loading experiment. In the elemental analysis data of the concentration series (Table S2 $\dagger$ ), slightly decreased carbon contents compared to the calculated values are found, whereas the nitrogen contents are slightly increased, supporting a decomposition of some BTC molecules. Moreover,

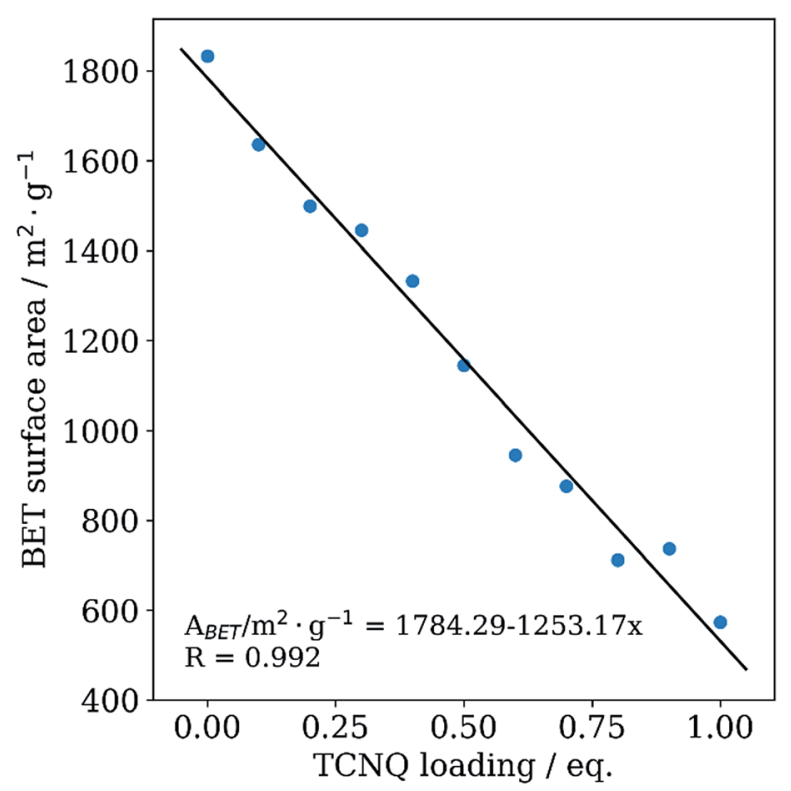

Fig. 3 BET surface area of $\mathrm{Cu}_{3} \mathrm{BTC}_{2}$ samples plotted against the TCNQ loading amount. Values of the linear fit are shown in the diagram. 


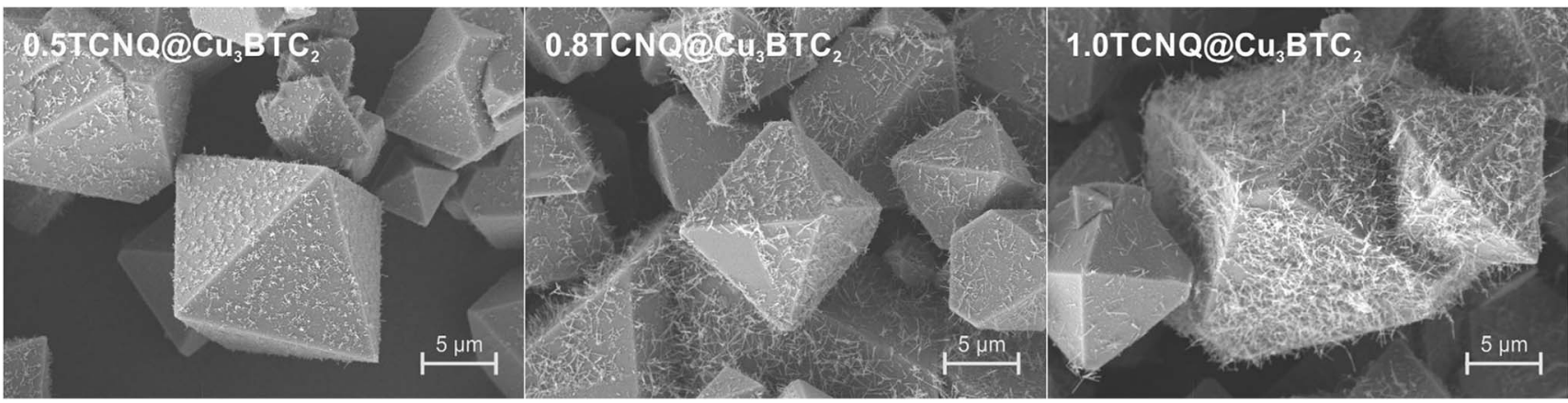

Fig. 4 SEM images of $\mathrm{Cu}_{3} \mathrm{BTC}_{2}$ loaded with 0.5 (left), 0.8 (middle), and 1.0 (right) equivalents of TCNQ. Cu(TCNQ) nanowires form on the surface of the octahedral $\mathrm{Cu}_{3} \mathrm{BTC}_{2}$ crystallites and their amount and dimensions increase with higher loading.

$\mathrm{Cu}_{3} \mathrm{BTC}_{2}$ is known to contain small quantities of $\mathrm{Cu}(\mathrm{I})$ species, which have been related to intrinsic defects and thermal treatment during activation. ${ }^{\mathbf{1 9}, 20}$ Surface sensitive XPS measurements (Fig. S19†) reveal a relatively high abundance of $\mathrm{Cu}(\mathrm{I})$ species at the surface of $\mathrm{Cu}_{3} \mathrm{BTC}_{2}$ crystallites. This $\mathrm{Cu}(\mathrm{I})$ concentration is further increased by the inevitable prolonged thermal treatment during the VPI process. The $\mathrm{Cu}(\mathrm{I})$ species, in turn, are able to reduce neutral TCNQ (via $\mathrm{Cu}(\mathrm{I}) \rightarrow \mathrm{Cu}(\mathrm{II})+\mathrm{e}^{-}$) and thus enable the formation of the surface impurity phase $\mathrm{Cu}(\mathrm{TCNQ})$. The presence of several TCNQ species (i.e. coordinated, uncoordinated, radical anion) is suggested by the broadening of the $\mathrm{N} 1 \mathrm{~s}$ signature in XPS (Fig. S20†). The formation of the $\mathrm{Cu}$ (TCNQ) byproduct could be reduced, but not fully inhibited, by using lower reaction temperatures (Fig. S15-S17 †). However, reduced temperature also compromise the ordering of TCNQ, as evidenced by the absence of the pronounced (111) reflection in PXRD (Fig. S2†).

The loading and coordination of TCNQ to open Cu sites can also be tracked by vibrational spectroscopy. The infrared (IR) spectra of pristine and TCNQ-loaded $\mathrm{Cu}_{3} \mathrm{BTC}_{2}$ are shown in Fig. 5. Whereas in pristine $\mathrm{Cu}_{3} \mathrm{BTC}_{2}$ a band in the $\mathrm{CN}$ vibration region is absent, a $\mathrm{CN}$ vibration mode at $2222 \mathrm{~cm}^{-1}$ appears in TCNQ-loaded samples and becomes more pronounced with higher TCNQ loading. Notably, a second vibrational mode at $2200 \mathrm{~cm}^{-1}$ and a shoulder at $2170 \mathrm{~cm}^{-1}$ appear for samples with high TCNQ loading ( $c f$. Fig. S6†). A red shift of the CN vibration was previously attributed to coordinated TCNQ involving a partial charge transfer. ${ }^{8,21}$ Due to the presence of the CuTCNQ byproduct in the samples, we here also assign some contribution to the signal at $2000 \mathrm{~cm}^{-1}$ and the shoulder at $2170 \mathrm{~cm}^{-1}$ to $\mathrm{Cu}(\mathrm{TCNQ})$, whose IR signature is known from the literature. ${ }^{22}$ The indication of multiple TCNQ species in the IR spectra matches well with the XPS signature in the $\mathrm{N} 1 \mathrm{~s}$ regime.

Thermogravimetric analysis (TGA) of 1.0TCNQ@ $\mathrm{Cu}_{3} \mathrm{BTC}_{2}$ (Fig. S7 $\dagger$ ) shows a decomposition that proceeds in one step with an onset at $300{ }^{\circ} \mathrm{C}$. This temperature is slightly lower than observed for pristine $\mathrm{Cu}_{3} \mathrm{BTC}_{2}$, which starts to decompose at $330^{\circ} \mathrm{C}$. The corresponding features in the differential scanning calorimetry (DSC) data are at $349.7{ }^{\circ} \mathrm{C}$ and $338.3{ }^{\circ} \mathrm{C}$ for the pristine and the loaded MOF, respectively. The 1.0TCNQ@ $\mathrm{Cu}_{3} \mathrm{BTC}_{2}$ sample shows an additional smaller peak at $314.2{ }^{\circ} \mathrm{C}$, indicating a step-wise decomposition.
Electrical transport measurements were performed on pressed pellets using an air-tight two-point-probe setup. $I-V$ curves were recorded from $-5 \mathrm{~V}$ to $5 \mathrm{~V}$ (Fig. S9†) and the conductivity $\sigma$ was calculated using eqn (1):

$$
\sigma=\frac{I}{V} \frac{d}{A}
$$

in which $I, V, d$ and $A$ are the measured electrical current, the applied voltage, the thickness of the pellet and the area of the pellet, respectively. The calculated room-temperature conductivities are plotted against the TCNQ loading (Fig. 6). We observe an exponential increase of the electrical conductivity upon TCNQ loading, starting from immeasurably small values for pristine $\mathrm{Cu}_{3} \mathrm{BTC}_{2}$ to $1.5 \times 10^{-4} \mathrm{~S} \mathrm{~cm}^{-1}$ for $1.0 \mathrm{TCNQ} @ \mathrm{Cu}_{3}-$ $\mathrm{BTC}_{2}$. This value is lower than the electrical conductivity reported for thin film samples infiltrated via the liquid phase $\left(0.07 \mathrm{~S} \mathrm{~cm}^{-1}\right){ }^{8}$ pointing at additional influence of grain boundaries and particle sizes as well as at the different measurement techniques used (pressed powder $v s$. thin film). ${ }^{23}$

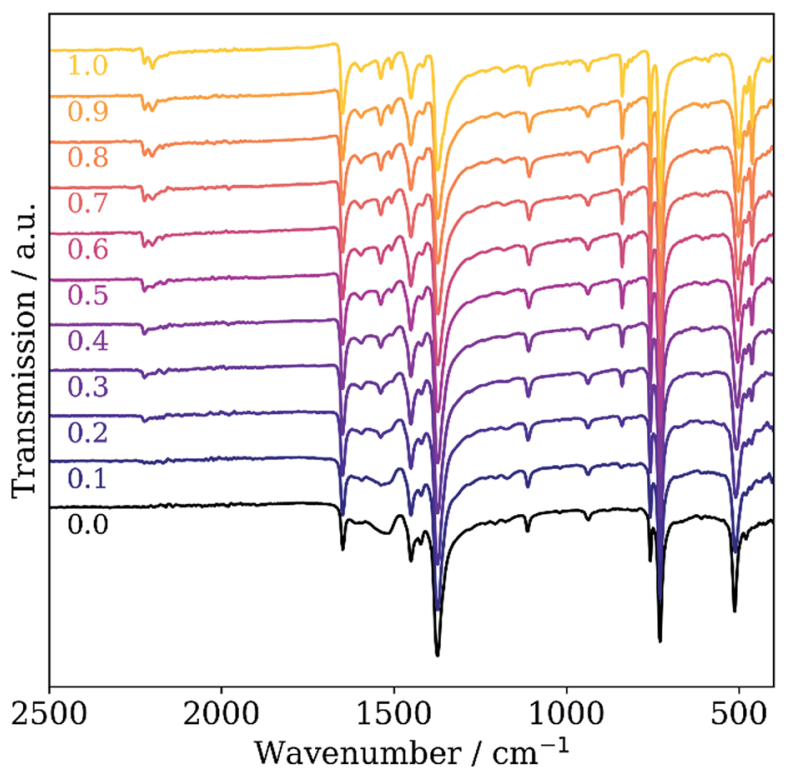

Fig. 5 IR spectra of pristine $\mathrm{Cu}_{3} \mathrm{BTC}_{2}$ (black) and the concentration series of $T C N Q$-loaded $\mathrm{Cu}_{3} \mathrm{BTC}_{2}$. TCNQ loadings are given below each spectrum as $x$ in $x \mathrm{TCNQ} \mathrm{aCu}_{3} \mathrm{BTC}_{2}$. 


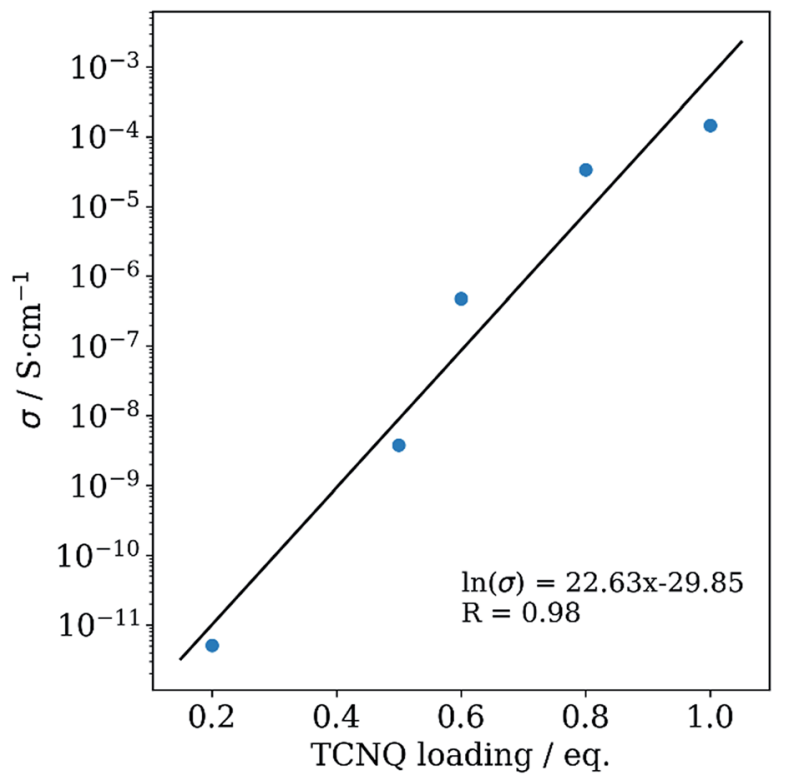

Fig. 6 Electrical conductivity of $\mathrm{TCNQ} \mathrm{aCu}_{3} \mathrm{BTC}_{2}$ samples plotted against their TCNQ loading amount.

The observed exponential increase of the electrical conductivity can be described by classical percolation theory. ${ }^{24,25}$ At low TCNQ loadings only few of the copper paddlewheel units are bridged by TCNQ and form localized conducting regions. With increasing TCNQ loading these bridged domains become interconnected and give rise to charge transport through the framework. Given the fact that TCNQ@ $\mathrm{Cu}_{3} \mathrm{BTC}_{2}$ samples in the literature showed high conductivities but no pronounced (111) reflection, ${ }^{8-10}$ disordered TCNQ might also contribute to the charge transport through the material.

The impact of the $\mathrm{Cu}(\mathrm{TCNQ})$ byproduct on the electrical transport, however, is intrinsically difficult to assess because $\mathrm{Cu}(\mathrm{TCNQ})$ can crystallize in two different phases. Phase II is a poor conductor, whereas phase $\mathrm{I}$ is an electrical semiconductor with a room temperature conductivity of $0.25 \mathrm{~S} \mathrm{~cm}^{-1}$. $^{18}$ Mechanical removal of the nanowires by sonication was unsuccessful and led to a disordering of the TCNQ molecules and a significant decrease in the conductivity (Fig. S3, S11 and S18†). A physical mixture of 1\% CuTCNQ/ $\mathrm{Cu}_{3} \mathrm{BTC}_{2}$ (estimated amount from SEM images) did not show any conductivity. In combination with previous findings in the literature for TCNQ@HKUST-1 in which $\mathrm{Cu}(\mathrm{TCNQ})$ as an impurity phase was not observed, we ascribe the increasing electrical conductivity as function of TCNQ loading to the formation of our host-guest system. Moreover, 1.0TCNQ@ $\mathrm{Cu}_{3} \mathrm{BTC}_{2}$ exhibits one of the highest-reported electrical conductivities paired with permanent porosity reported to date, comparing with other conductive MOFs of similar porosities, such as $\mathrm{Cd}_{2}$ (TTFTB). ${ }^{6,26}$ The synthesis of a reference sample of $x \mathrm{TCNQ}_{0} \mathrm{Cu}_{3} \mathrm{BTC}_{2}(x=0.4$ as determined by $\mathrm{EA})$ via liquid phase infiltration revealed no indication of TCNQ ordering, but a conductivity in the order of $10^{-1} \mathrm{~S} \mathrm{~cm}^{-1}$, which is significantly higher than for samples with comparable TCNQ loading prepared via VPI. However, this sample shows a non- ohmic behavior (deviation from the linear $I-V$ curve) at high potentials that we assign to electrochemical processes of water/ solvent inside the pores (Fig. S21-S24†). This finding emphasizes the importance of solvent exclusion during the guest infiltration step and highlights the need for in-depth structural and spectroscopic studies of Guest@MOF systems to develop and validate structure-property relationships.

\section{Conclusions}

We developed an optimized VPI strategy under thermodynamic control and used it to introduce stoichiometric amounts of TCNQ to obtain a concentration series of $x \mathrm{TCNQ}_{0} \mathrm{Cu}_{3} \mathrm{BTC}_{2}$ with $0 \leq x \leq 1.0$ under strict inert conditions. No washing step is needed to remove excessive TCNQ, which is advantageous over the kinetically controlled loading procedure presented in previous studies. ${ }^{8-10,16}$ High reaction temperatures and long exposure times evidently promote an ordered, periodic arrangement of TCNQ within the (111) crystal lattice plane and the bridging coordination motif of TCNQ and the $\mathrm{Cu}$ atoms of two neighbouring paddle-wheel units. To our knowledge this is the first crystallographic evidence for the integration of TCNQ into the framework of $\mathrm{Cu}_{3} \mathrm{BTC}_{2}$, and further demonstrates that the introduction of a non-innocent guest molecule can be regarded as a new element of MOF property design. Additional evidence supporting the accommodation of TCNQ within the pores of the framework is the systematic decrease in the BET surface area. SEM images of the infiltrated samples show the formation of nanowires on the MOF crystal surface during VPI that were identified as $\mathrm{Cu}(\mathrm{TCNQ})$. Their formation can be reduced but not entirely suppressed at lower temperatures. IR data recorded during this work shows two nitrile vibration modes that can be attributed to TCNQ molecules inside the $\mathrm{Cu}_{3} \mathrm{BTC}_{2}$ framework and to the TCNQ anion in $\mathrm{Cu}(\mathrm{TCNQ})$. The electrical conductivity of the concentration series increases exponentially with the amount of TCNQ used in the VPI. Even though, a quantitative analysis of the contribution of the hostguest complex and the $\mathrm{Cu}(\mathrm{TCNQ})$ byproduct to the electrical conductivity of the material is difficult, the obtained material shows high conductivity values accompanied by high permanent porosity. In fact, the BET surface area of 1.0TCNQ@ $\mathrm{Cu}_{3} \mathrm{BTC}_{2}$ is among the highest for electrically conductive MOFs. ${ }^{6,26}$ The combination of these two properties creates a high potential for using this material in sensing or electronic device fabrication where access to the pores provides an essential new function. In this respect, well defined, oriented $\mathrm{Cu}_{3} \mathrm{BTC}_{2}$ thin films deposited and infiltrated with TCNQ using solvent-free vapor phase techniques represent the ultimate goal. Pointing to the complexity of this system, further detailed analysis is required, beyond standard characterization techniques, to fully understand the ordering of TCNQ and the conductivity mechanism.

\section{Experimental}

\section{General information}

All chemicals were purchased from commercial suppliers (ABCR, Acros Organics, Alfa Aesar, Sigma Aldrich) and used 
without further purification unless otherwise stated. Solvents used for the synthesis and washing steps were reagent grade or higher.

\section{Vapor phase infiltration}

$\mathrm{Cu}_{3} \mathrm{BTC}_{2}$ was synthesized following the literature procedure. ${ }^{27}$ The tile crystalline product was continuously washed with ethanol and dichloromethane in a Soxhlet apparatus for one week, respectively, to rigorously remove all reactants and highboiling solvents. The washing solution was replaced by the respective fresh solvent once. Subsequently, the blue powder was desolvated under high vacuum $\left(\sim 10^{-6} \mathrm{mbar}\right)$ at $180^{\circ} \mathrm{C}$ to yield pristine $\mathrm{Cu}_{3} \mathrm{BTC}_{2}$. TCNQ was recrystallized three times from acetonitrile under inert conditions to yield molecular, crystalline TCNQ in high purity.

Inside an argon glovebox, $200 \mathrm{mg}$ of $\mathrm{Cu}_{3} \mathrm{BTC}_{2}$ and distinct amounts of TCNQ were physically mixed and filled into borosilicate glass tubes to prepare a concentration series of $x \mathrm{TCNQ}_{3} \mathrm{Cu}_{3} \mathrm{BTC}_{2}\left(x=n(\mathrm{TCNQ}) / n\left(\mathrm{Cu}_{3} \mathrm{BTC}_{2}\right)\right.$ with $x=0.1$, $0.2, \ldots, 1.0)$. The glass tube was then evacuated $\left(\sim 10^{-3} \mathrm{mbar}\right)$ and flame sealed to give a closed system for the VPI process. The sealed ampules were placed inside a convection oven at $180{ }^{\circ} \mathrm{C}$ for 72 hours allowing the TCNQ to sublime and diffuse into the MOF. After cooling down to room temperature the ampules were transferred into the glovebox and stored for further characterization.

\section{Powder X-ray diffraction}

Powder X-ray diffraction (PXRD) data was collected in a $2 \theta$ range of $5-50^{\circ}$ in steps of $0.0016413^{\circ}(2 \theta)$ on a PANalytical Empyrean equipped with a $\mathrm{Cu}$ X-ray tube operated at $45 \mathrm{kV}$ and $40 \mathrm{~mA}$. The samples were filled into borosilicate capillaries of $0.7 \mathrm{~mm}$ diameter and mounted onto a capillary spinner. The radiation was focused onto the sample through a focusing X-ray beam mirror equipped with a $1 / 8^{\circ}$ divergence slit and 0.02 radian soller slits. The diffracted beam was detected by a PIXcel1D detector in receiving slit mode equipped with a $1 / 8^{\circ}$ anti-scatter slit and 0.02 radian soller slits.

\section{Thermogravimetric analysis/differential scanning calorimetry}

TGA/DSC measurements were carried out on a Mettler Toledo TGA/DSC 1 equipped with an auto sampler unit. Aluminum crucibles $(100 \mu \mathrm{L})$ were filled with $5-10 \mathrm{mg}$ of MOF powder inside the glovebox and tightly capped with an aluminum lit. The crucible was transferred into the TGA chamber and a hole was pinched into the lit while the stream of $\operatorname{Ar}\left(40 \mathrm{~mL} \mathrm{~min}{ }^{-1}\right)$ was already on. The temperature was ramped from $30{ }^{\circ} \mathrm{C}$ to $550{ }^{\circ} \mathrm{C}$ at a rate of $5^{\circ} \mathrm{C} \mathrm{min}^{-1}$. The instrument was calibrated to a blank sample prior to the measurements.

\section{Porosimetry measurements}

Porosimetry measurements of pristine and guest-loaded MOF powders were performed using a Micromeritics 3 flex to determine their BET surface area. Therefore, approximately $60 \mathrm{mg}$ of a sample was filled into a BET tube and evacuated for $3 \mathrm{~h}$ at room temperature prior to the measurement. Nitrogen isotherms were recorded at $77 \mathrm{~K}$. The BET surface area was calculated from data points in the relative pressure range of 0.01 to 0.1 .

\section{Fourier transform infrared spectroscopy}

Fourier transform infrared spectroscopy (FTIR) of powder samples was done in argon atmosphere on an ALPHA FTIR spectrometer (Bruker) equipped with a Pt attenuated total reflectance (ATR) unit at room temperature in the range of 400$4000 \mathrm{~cm}^{-1}$ with a resolution of $2 \mathrm{~cm}^{-1} .64$ scans were recorded per measurement.

\section{Elemental analysis}

Elemental analysis (EA) for the elements C, H, N, and S was carried out on a Hekatech EuroEA Elementaranalysator. $\mathrm{Cu}$ contents were determined by atom absorption spectroscopy (AAS) after decomposing the sample in a mixture of sulfuric acid and nitric acid in a CEM microwave.

\section{Scanning electron microscopy}

Top-view micrographs of MOF powders were conducted on a Merlin high-resolution scanning electron microscope (Carl Zeiss) using an acceleration voltage of $5 \mathrm{kV}$. The samples were transferred from a glove box into the analysis chamber under argon atmosphere using a transfer vessel (Leica EM VC500). To avoid charging, the sample was coated with $5 \mathrm{~nm}$ of Pt prior to the measurement using a Leica EM ACE600. Energy dispersive $\mathrm{X}$-ray spectroscopy (EDX) analysis was conducted using an XMAX EXTREME EDX detector (Oxford Instruments). Measurements were carried out by application of an acceleration voltage of $5 \mathrm{kV}$ and a probing current of $100 \mathrm{pA}$ for SEM and 1000 pA for EDX.

\section{Auger electron spectroscopy}

AES measurements were performed at room temperature with a scanning Auger electron spectroscope (JEOL Ltd. JAMP-9500F field emission scanning Auger microprobe) system. Samples were prepared by spreading powder particles over a gold-coated surface. AES spectra were acquired with a primary beam of 10 $\mathrm{keV}$. The take-off angle of the instrument was $0^{\circ}$. The differential energy spectrum was used to subtract background from the direct Auger spectrum for calculating the band-to-band intensity. The first differential $\mathrm{d}(N(E)) / \mathrm{d}(E)$ Auger spectra were obtained by numerical derivation of the direct $N(E)$ integrated Auger data displaying an absolute scale with counts/second units by a universal Savitzky-Golay (SG) quadratic differential filter using seven points and used to calculate the band-to-band intensity of Auger electrons and derive the elemental compositions. The differential spectrum is simply the differential of the direct spectrum with respect to energy. The spectra were calibrated with the carbon band at $263.0 \mathrm{eV}$. For Auger elemental analysis an $8 \mathrm{~nm}$ probe diameter was used. Elemental mapping was analysed by AES. Elemental images were acquired with a primary beam of $10 \mathrm{keV}$. The take-off angle of the instrument was $0^{\circ}$. The coloured images are elemental 
distribution maps over the area shown in the upper right image (magnification $80000 \times$ ).

\section{X-ray photoelectron spectroscopy}

XPS was carried out using a PHI5000 Versa Probe II (Physical Electronics $\mathrm{GmbH}$ ) with an $\mathrm{Al}$ anode. To avoid air exposure, powder samples were transferred from an argon-filled glovebox to the analysis chamber using a transfer vessel filled with argon gas. The probed surface area was $100 \mu \mathrm{m} \times 1400 \mu \mathrm{m}$ (i.e., X-ray spot size) and an X-ray power of $100 \mathrm{~W}$ was used. The pass energy of the analyzer was set to $23.5 \mathrm{eV}$ for detail spectra and to $187.9 \mathrm{eV}$ for survey scans. The chamber pressure was in the range of $10^{-7} \mathrm{~Pa}$ during the measurements. All spectra were charge corrected to a binding energy of $284.8 \mathrm{eV}$ for the $\mathrm{C} 1 \mathrm{~s}$ line corresponding to adventitious aliphatic carbon. Measurements were evaluated using CasaXPS V2.3.17 software.

\section{Electrical conductivity measurements}

Inside an Ar filled glovebox, MOF powders ( $\sim 70 \mathrm{mg})$ were filled into an air-tight press cell and compressed uniaxially at $3 \mathrm{t}$ (375 MPa) for 2 minutes. The press cell was mounted into an aluminium frame and fixed with a screw applying a torque of $10 \mathrm{Nm}$ ( $c f$. ESI Fig. $8 \dagger) .{ }^{28} I-V$ curves were recorded between the two stainless steel electrodes of the press cell using an EC-Lab Electrochemistry SP-300 potentiostat/galvanostat (Bio-Logic Science Instruments). Conductivity measurements were carried out at $298 \mathrm{~K}$ (climate chamber, Weiss Klimatechnik, $1 \mathrm{~h}$ equilibration time) sweeping the voltage between $5 \mathrm{~V}$ and $-5 \mathrm{~V}$ at a scan rate of $100 \mathrm{mV} \mathrm{s}^{-1}$.

\section{Conflicts of interest}

There are no conflicts to declare.

\section{Acknowledgements}

We thank Dr Vitalie Stavila and Dr Andrew Ullman for helpful discussions and Pia Vervoorts for help with BET measurements. Funding for this project was provided by the Sandia Laboratory Directed Research and Development (LDRD) Program. Sandia National Laboratories is a multimission laboratory managed and operated by National Technology and Engineering Solutions of Sandia, LLC, a wholly owned subsidiary of Honeywell International, Inc., for the U.S. Department of Energy's National Nuclear Security Administration under contract DE-NA0003525. CS acknowledges his scholarships from the German Academic Scholarship Foundation and from the German Chemical Industry Fund (FCI). RK acknowledges his scholarship from the FCI. This project was further supported by a PPP travel grant from the German Academic Exchange Service (DAAD).

\section{Notes and references}

1 I. Stassen, N. Burtch, A. Talin, P. Falcaro, M. Allendorf and R. Ameloot, Chem. Soc. Rev., 2017, 46, 3185-3241.
2 V. Stavila, A. A. Talin and M. D. Allendorf, Chem. Soc. Rev., 2014, 43, 5994-6010.

3 G. Maurin, C. Serre, A. Cooper and G. Ferey, Chem. Soc. Rev., 2017, 46, 3104-3107.

4 O. M. Yaghi, M. O'Keeffe, N. W. Ockwig, H. K. Chae, M. Eddaoudi and J. Kim, Nature, 2003, 423, 705-714.

5 M. Eddaoudi, J. Kim, N. Rosi, D. Vodak, J. Wachter, M. O'Keeffe and O. M. Yaghi, Science, 2002, 295, 469-472.

6 L. Sun, M. G. Campbell and M. Dincă, Angew. Chem., Int. Ed., 2016, 55, 3566-3579.

7 L. Sun, C. H. Hendon, S. S. Park, Y. Tulchinsky, R. Wan, F. Wang, A. Walsh and M. Dinca, Chem. Sci., 2017, 8, 44504457.

8 A. A. Talin, A. Centrone, A. C. Ford, M. E. Foster, V. Stavila, P. Haney, R. A. Kinney, V. Szalai, F. El Gabaly, H. P. Yoon, F. Léonard and M. D. Allendorf, Science, 2014, 343, 66-69.

9 X. Chen, Z. Wang, Z. M. Hassan, P. Lin, K. Zhang, H. Baumgart and E. Redel, ECS J. Solid State Sci. Technol., 2017, 6, 150-153.

10 T. Neumann, J. Liu, T. Wächter, P. Friederich, F. Symalla, A. Welle, V. Mugnaini, V. Meded, M. Zharnikov, C. Wöll and W. Wenzel, ACS Nano, 2016, 10, 7085-7093.

11 C. H. Hendon and A. Walsh, Chem. Sci., 2015, 6, 3674-3683. 12 K. J. Erickson, F. Léonard, V. Stavila, M. E. Foster, C. D. Spataru, R. E. Jones, B. M. Foley, P. E. Hopkins, M. D. Allendorf and A. A. Talin, Adv. Mater., 2015, 27, 3453-3459.

13 M. D. Allendorf, M. E. Foster, F. Léonard, V. Stavila, P. L. Feng, F. P. Doty, K. Leong, E. Y. Ma, S. R. Johnston and A. A. Talin, J. Phys. Chem. Lett., 2015, 6, 1182-1195.

14 W. Kaim and M. Moscherosch, Coord. Chem. Rev., 1994, 129, 157-193.

15 M. E. Peover, J. Chem. Soc., Faraday Trans., 1964, 60, 479483.

16 P. M. Usov, H. Jiang, H. Chevreau, V. K. Peterson, C. F. Leong and D. M. D'Alessandro, J. Phys. Chem. C, 2017, 121, 2633026339.

17 K. Schlichte, T. Kratzke and S. Kaskel, Microporous Mesoporous Mater., 2004, 73, 81-88.

18 R. A. Heintz, H. Zhao, X. Ouyang, G. Grandinetti, J. Cowen and K. R. Dunbar, Inorg. Chem., 1999, 38, 144-156.

19 N. Nijem, H. Bluhm, M. L. Ng, M. Kunz, S. R. Leone and M. K. Gilles, Chem. Commun., 2014, 50, 10144-10147.

20 J. Szanyi, M. Daturi, G. Clet, D. R. Baer and C. H. F. Peden, Phys. Chem. Chem. Phys., 2012, 14, 4383-4390.

21 J. S. Chappell, A. N. Bloch, W. A. Bryden, M. Maxfield, T. O. Poehler and D. O. Cowan, J. Am. Chem. Soc., 1981, 103, 2442-2443.

22 M. Inoue and M. B. Inoue, J. Chem. Soc., Faraday Trans. 2, 1985, 81, 539-547.

23 L. Sun, S. S. Park, D. Sheberla and M. Dincă, J. Am. Chem. Soc., 2016, 138, 14772-14782.

24 B. Abeles, H. L. Pinch and J. I. Gittleman, Phys. Rev. Lett., 1975, 35, 247-250.

25 B. J. Last and D. J. Thouless, Phys. Rev. Lett., 1971, 27, 17191721. 
26 M. L. Aubrey, B. M. Wiers, S. C. Andrews, T. Sakurai, S. E. Reyes-Lillo, S. M. Hamed, C.-J. Yu, L. E. Darago, J. A. Mason, J.-O. Baeg, F. Grandjean, G. J. Long, S. Seki, J. B. Neaton, P. Yang and J. R. Long, Nat. Mater., 2018, 17, 625-632.
27 M. K. Bhunia, J. T. Hughes, J. C. Fettinger and A. Navrotsky, Langmuir, 2013, 29, 8140-8145.

28 W. Zhang, D. A. Weber, H. Weigand, T. Arlt, I. Manke, D. Schröder, R. Koerver, T. Leichtweiss, P. Hartmann, W. G. Zeier and J. Janek, ACS Appl. Mater. Interfaces, 2017, 9, 17835-17845. 